\title{
LOGARITHMIC SEQUENCES
}

JOSEPH MILKMAN

Introduction. M. Augustin-Louis Cauchy in his famous Cours d'Analyse de l'ecole Royale Polytechnique (Part I, Analyse Algébrique, Chapter 5, page 109), published in 1821, gives a beautiful proof that the only continuous solution of the functional equation $f(x)+f(y)$ $=f(x y)$, where $f(x)$ is defined for all real numbers $x$, is the function $f(x)=a \ln x$. Cauchy's proof reduces the equation to the Cauchy equation $f(x)+f(y)=f(x+y)$. In 1905 G. Hamel in the Mathematische Annalen proved that the discontinuous solutions of Cauchy's equation are totally discontinuous. In 1919 in the Transactions of the American Mathematical Society B. Blumberg proved that the only measurable function satisfying Cauchy's equation is the function $A x$, hence the only measurable solution of $f(x)+f(y)=f(x y)$ is $a \ln x$. In the past thirty-five years there have been a number of papers on these functional equations where the domain of the independent variable has usually been the real number system. In $1946 \mathrm{P}$. Erdos proved by analytic number theory techniques that if $f(m)$ is additive and $f(m+1) \geqq f(m)$ then $f(m)=c \ln m$ (Ann. of Math. vol. 47 (1946)). This implies "If $f(m+1)>f(m)$ and $f(m)+f(n)=f(m n)$ hold for all positive integers $m$ and $n$, then $f(m)=c \ln m$," a result for which the author obtained a more elementary proof in 1950 (Proc. Amer. Math. Soc. vol. 1 , no. 4). In a problem of designing a computating mechanism the author considered the monotone solutions of $f(x)+f(y)$ $=f(x y)$ where the domain of the independent variable is the finite set of integers $1,2, \cdots, n$. This led him to the notion of logarithmic sequences, the subject of this paper.

Definition. Logarithmic Sequence. A logarithmic sequence is a set of $n$ real numbers $a_{i}, i=1,2, \cdots, n$ such that

(1) $0 \leqq a_{i} \leqq 1$,

(2) $a_{k}+a_{l}<a_{r}+a_{s}$ if $k l<r s$,

(3) $a_{k}+a_{l}=a_{r}+a_{8}$ if $k l=r s$,

$n$ is called the order of the logarithmic sequence.

Notation. $p_{i}$ : For $r \leqq n$ and $s \leqq n$ form all the different products $r s$. The sequence $p_{1}, p_{2}, p_{3}, \cdots, p_{t}$ is the sequence of all these distinct products arranged in increasing order of magnitude.

$s_{i}$ : Corresponding to the logarithmic sequence $a_{j}, j=1,2, \cdots, n$ and $p_{i}=r s, i=1,2, \cdots, t$ we define $s_{i}=a_{r}+a_{s}$.

Presented to the Society April 16, 1955; received by the editors March 24, 1955 and, in revised form, February 24, 1956. 
The $s_{i}$ are monotonically increasing.
$\Delta_{i}$ :
$\Delta_{i}=s_{i+1}-s_{i}>0$
for $i=1,2, \cdots, t-1$.

$\operatorname{Min} \Delta(\mathrm{a})$ denotes the smallest $\Delta_{i}$ for a given logarithmic sequence $a_{i}$.

Definition. Maximizing Sequence. $m_{1}, m_{2}, \cdots, m_{n}$ is a maximizing sequence if it is a logarithmic sequence and $\min \Delta(m) \geqq \min \Delta(a)$ for all logarithmic sequences $a_{1}, a_{2}, \cdots, a_{n}$.

PROPERTIES OF LOGARITHMIC SEQUENCES OF ORDER $n$.

1. $a_{1}<a_{2}<a_{3} \cdots<a_{n}$ for $p_{i}=i$ when $i=1,2, \cdots, n$ and $s_{i}$ $=a_{1}+a_{i}$ by definition $a_{1}+a_{1}<a_{1}+a_{2}<\cdots<a_{1}+a_{n}$.

2. $\min \Delta(a) \leqq\left(2-s_{1}\right) /(t-1)$ for $s_{t}>s_{i}$ for $i=1,2, \cdots, t-1$,

$$
\begin{aligned}
s_{t} & =a_{n}+a_{n} \leqq 1+1=2, \\
s_{i} & >s_{1}=a_{1}+a_{1} \geqq 0 \\
2 & \geqq s_{t}=s_{1}+\Delta_{1}+\Delta_{2}+\cdots+\Delta_{t-1},
\end{aligned}
$$

3. $\min \Delta(a) \leqq\left(s_{p}-s_{q}\right) /(p-q)$.

4. If $a_{1}, a_{2}, \cdots, a_{k}, \cdots, a_{n}$ is a logarithmic sequence of order $n$ then $a_{1}, a_{2}, \cdots, a_{k}$ is a logarithmic sequence of order $k$.

5. $\Delta_{1}>\Delta_{2}>\Delta_{3}>\cdots>\Delta_{n-1}$.

PROOF. For $k<n$ there is an $r$ such that $2 a_{k}=s_{r}$ and

$$
\begin{aligned}
& a_{k+1}+a_{k-1}=s_{r-1}, \\
& 2 a_{k}>a_{k+1}+a_{k-1}, \\
& \Delta_{k-1}>\Delta_{k} .
\end{aligned}
$$

6. If $p_{i} p_{k}=p_{r} p_{j}=$ some $p_{s}$ then $s_{i}-s_{j}=s_{r}-s_{k}=\Delta_{j}+\Delta_{j+1} \ldots$ $+\Delta_{i-1}$.

7. If $n$ is a prime $t(n)=t(n-1)+n$.

8. $\Delta_{t-1}=\Delta_{t-2}=\Delta_{n-1}$ for $s_{t}=2 a_{n}, s_{t-1}=a_{n}+a_{n-1}$ and $s_{t-2}=2 a_{n-1}$.

9. In the system of linear inequalities $\Delta_{i}>0$ for $i=1,2, \cdots, t-1$, the inequalities $i=1,2, \cdots, n-1$ are superfluous.

Proof. If $i<n$ let $p_{k}=2 i$ and $p_{l}=2(i+1)$ then $s_{k}=a_{2}+a_{i}$ and $s_{l}=a_{2}+a_{i+1}, \quad s_{l}-s_{k}=a_{i+1}-a_{i}=\Delta_{i}=\Delta_{k}+\Delta_{k+1}+\cdots+\Delta_{l-1}$. Hence if

$$
n / 2 \leqq i<n, \quad \Delta_{i}=\Delta_{k}+\Delta_{k+1}+\cdots+\Delta_{l-1}, \quad k \geqq n
$$
if

$$
n / 4 \leqq i<n / 2, \quad \Delta_{i}=\Delta_{k}+\Delta_{k+1}+\cdots+\Delta_{l-1}, \quad k \geqq n / 2
$$


if

$$
n / 2^{n} \leqq i<n / 2^{n-1}, \quad \Delta_{i}=\Delta_{k}+\Delta_{k+1}+\cdots+\Delta_{l-1}, \quad k \geqq n / 2^{n-1} .
$$

10. Let the logarithmic sequence $a_{1}, a_{2}, \cdots, a_{n}$ be represented by the position vector $a \equiv\left(a_{1}, \cdots, a_{n}\right)$ in $n$-dimensional Euclidean space. If $a$ and $b$ represent logarithmic sequences then $k a$ $\equiv\left(k a_{1}, k a_{2}, \cdots, k a_{n}\right)$ for $0<k<1$ and $(m a+n b) /(m+n)$ for all $m>0$ and $n>0$ represent logarithmic sequences. The set of points $L$ representing logarithmic sequences is convex, connected and dense in itself.

11. If $p_{1}, p_{2}, p_{3}, \cdots, p_{k}$ are all the primes less than or equal to $n$, then $a_{1}, a_{p_{1}}, a_{p_{2}}, \cdots, a_{p_{k}}$ determine the logarithmic sequence $a_{1}, a_{2}, a_{3}, \cdots, a_{n}$.

12. If

$$
a \equiv\left|\begin{array}{c}
a_{2} \\
a_{3} \\
\vdots \\
a_{n}
\end{array}\right|, \quad \Delta \equiv\left|\begin{array}{c}
\Delta_{1} \\
\Delta_{2} \\
\vdots \\
\Delta_{n-1}
\end{array}\right|, \quad a_{1}=0
$$

and

$$
A \equiv\left|\begin{array}{ccccccc}
1 & 0 & 0 & . & . & . & 0 \\
1 & 1 & 0 & . & . & . & 0 \\
1 & 1 & 1 & . & . & . & 0 \\
. & . & . & . & . & . & . \\
. & . & . & . & . & . & . \\
. & . & . & . & . & . & . \\
1 & 1 & 1 & . & . & 1 & 0 \\
1 & 1 & 1 & . & . & 1 & 1
\end{array}\right|
$$

then in matrix notation $a=A \Delta$ and $\Delta=A^{-1} a$.

13. If $p_{1}, p_{2}, \cdots, p_{k}$ are the primes $\leqq n, a_{1}=0$ and $s_{i}=r_{1_{i}} a_{p_{1}}$ $+r_{2 i} a_{p_{2}}+\cdots+r_{k i} a_{p_{k}}$ for $i=1,2, \cdots, t-1$ then the rank of the matrix

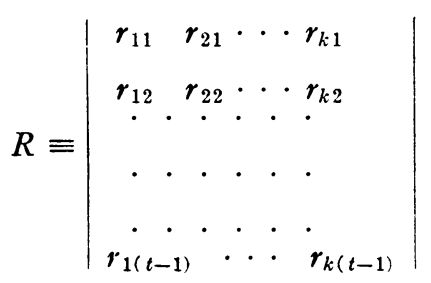


is $k$ and

$$
\Delta \equiv\left|\begin{array}{l}
\Delta_{1} \\
\Delta_{2} \\
\vdots \\
\Delta_{t-1}
\end{array}\right|=A^{-1} R\left|\begin{array}{l}
a_{p_{1}} \\
a_{p_{2}} \\
\vdots \\
a_{p_{k}}
\end{array}\right|
$$

where $A^{-1} R$ has rank $k$.

Properties of MAXIMIZING Sequences $m_{1}, m_{2}, \cdots, m_{n}$.

1. $m_{1}=0$ and $m_{n}=1$.

Proof. Assume $m_{n}<1$ and let $\lambda=1 / m_{n}>1$.

The sequence $\lambda m$ is a logarithmic sequence.

$$
\begin{aligned}
s_{i}(\lambda m) & =\lambda s_{i}(m), \\
\Delta_{i}(\lambda m) & =\lambda \Delta_{i}(m) \quad \text { for } i=1,2, \cdots, t-1, \\
\min \Delta(\lambda m) & =\lambda \min \Delta(m)>\min \Delta(m)
\end{aligned}
$$

contrary to the assumption that $m$ was a maximizing sequence. Hence $m_{n}=1$.

$$
\begin{aligned}
0 & \leqq m_{i}-m_{1} \leqq 1, \\
\left(m_{k}-m_{1}\right)+\left(m_{l}-m_{1}\right) & <\left(m_{r}-m_{1}\right)+\left(m_{s}-m_{1}\right) \text { when } k l<r s, \\
\left(m_{k}-m_{1}\right)+\left(m_{l}-m_{1}\right) & =\left(m_{r}-m_{1}\right)+\left(m_{s}-m_{1}\right) \text { when } k l=r s .
\end{aligned}
$$

Therefore $m_{i}-m_{1}$ is a logarithmic sequence.

$$
\begin{aligned}
s_{i}\left(m-m_{1}\right) & =s_{i}(m)-2 m_{1}, \\
\Delta s_{i}\left(m-m_{1}\right) & =\Delta s_{i}(m), \\
\min \Delta\left(m-m_{1}\right) & =\min \Delta(m) \text { or } m-m_{1}
\end{aligned}
$$

is a maximizing sequence and $m_{n}-m_{1}=1=m_{n}$. Hence $m_{1}=0$.

2. $\Delta_{1}=\Delta_{2}+\Delta_{3}$ for $\Delta_{1}=s_{2}$ and $s_{4}=2 s_{2}$.

3. $\sum_{1}^{t-1} \Delta_{i}=2, \sum_{1}^{m-1} \Delta_{i}=1$ and hence $\sum_{n}^{t-1} \Delta_{i}=1$.

4. $\min \Delta(m)$ of a maximizing sequence of order $n \leqq \min \Delta(m)$ of a maximizing sequence of order $k$ if $n>k$. This is a consequence of property 4 of logarithmic sequences.

5. If $a_{1}, a_{2}, \cdots, a_{n}$ and $b_{1}, b_{2}, \cdots, b_{n}$ are maximizing sequences and $\Delta_{k}(a)=\min \Delta(a)$, then $\Delta_{k}(a)=\Delta_{k}(b)$ for at least one $k$ for which $\Delta_{k}(a)=\min \Delta(a)$.

Proof. $\Delta_{i}(a+b) / 2=\left(\Delta_{i}(a)+\Delta_{i}(b)\right) / 2>\Delta_{k}(a)$ for all $i$ except those for which $\Delta_{i}(a)=\Delta_{i}(b)=\Delta_{k}(a)$. The inequality cannot hold for all $i$ since $a$ is a maximizing sequence. Hence there must be a $k$ such that

$$
\Delta_{k}(a)=\Delta_{k}(b)=\min \Delta(a)=\min \Delta(b) .
$$


THEOREM I. A maximizing sequence of order $n$ exists for every natural number $n$.

Proof. Let $L$ be the set of points $a \equiv\left(a_{1}, a_{2}, \cdots, a_{n}\right)$ in $n$ dimensional Euclidean space for which $a$ is a logarithmic sequence. The set $L$ is bounded since it lies inside the rectangle $0 \leqq x_{i} \leqq 1$. Let $\bar{L}$ be the closure of $L$. If $x \equiv\left(x_{1}, x_{2}, \cdots, x_{n}\right)$ is in $\bar{L}$ then $s_{i}(x)=x_{r}+x_{s}$ (when $\left.p_{i}=r s\right)$ is a continuous function of $x$ in $\bar{L}$ for $i=1,2, \cdots, t . \Delta_{i}(x)$ $=s_{i+1}(x)-s_{i}(x)$ is a continuous function of $x$ in $\bar{L}$ for $i=1,2, \cdots$, $t-1$. Let $\min \Delta(x)$ be the minimum of $\Delta_{i}(x)$ for fixed $x$ and variable $i$. Min $\Delta(x)$ is a continuous function of $x$ on $\bar{L}$, a closed bounded set. Hence there is a point $m$ in $\bar{L}$ at which $\min \Delta(x)$ is a maximum. We will show that $m$ is in $L$ and hence is a maximizing sequence.

$m$ is a limit point of logarithmic sequences. There exists a sequence of points, belonging to $L, l^{i} \equiv\left(l_{1}^{t}, l_{2}^{t}, \cdots, l_{n}^{l}\right)$ with $m$ as a limit.

$$
\begin{aligned}
& 0 \leqq l_{1}^{i}<l_{2}^{i}<\cdots<l_{n}^{i} \leqq 1 \text { for } i=1,2,3, \cdots, \\
& 0 \leqq m_{1} \leqq m_{2} \leqq \cdots \leqq m_{n} \leqq 1 .
\end{aligned}
$$

Suppose $m_{r}=m_{r+1}$ then $s_{r}(m)=m_{r}+m_{1}$ and $s_{r+1}(m)=m_{r+1}+m_{1}$. $\Delta_{r}(m)=0$ and hence $\min \Delta(m) \leqq 0$ contrary to $m$ being a maximum point for $\min \Delta(m)$ since $\log _{n} i, i=1,2, \cdots, n$ is a logarithmic sequence with $\min \Delta>0$. Therefore

$$
\begin{aligned}
0 & \leqq m_{1}<m_{2}<m_{3}<\cdots<m_{n} . \\
l_{k}^{i}+l_{l}^{i} & <l_{r}^{i}+l_{s}^{i} \text { if } k l<r s .
\end{aligned}
$$

Hence $m_{k}+m_{l} \leqq m_{r}+m_{s}$ if $k l<r s$ or $s_{1}(m) \leqq s_{2}(m) \leqq s_{3}(m) \leqq \cdots$ $\leqq s_{t}(m)$. Suppose $s_{j}(m)=s_{j+1}(m)$ then $\Delta_{j}(m)=0$ and $\min \Delta(m) \leqq 0$ which is impossible. Therefore $m_{k}+m_{l}<m_{r}+m_{s}$ if $k l<r s$. Finally $l_{k}^{t}+l_{l}^{l}=l_{r}^{l}+l_{s}^{t}$ if $k l=r s$ implies $m_{k}+m_{l}=m_{r}+m_{s}$ if $k l=r s$ and hence $m$ is a logarithmic sequence and therefore a maximizing sequence.

Lemma. A maximum minimum point of a finite set of hyperplanes can be determined in a finite number of operations, if the set of hyperplanes has a maximum minimum point. Furthermore the maximum minimum point lies in a level intersection of a subset of the hyperplanes.

Proor. Let $L_{i} \equiv \sum_{j=1}^{p} a_{i}^{j} x_{j}+c_{i}, i=1,2, \cdots, n$ be $n$ linear functions of the $p$ variables $x_{1}, x_{2}, \cdots, x_{p}$ or the point $X \equiv\left(x_{1}, x_{2}, \cdots, x_{p}\right)$ in $p$-dimensional Euclidean space. $M$ in $L_{i}(X)$ denotes the function of $X$ whose value is the smallest number of the set $L_{1}(X), L_{2}(X), \cdots$, $L_{n}(X)$. We wish to determine a point $X$ for which $\operatorname{Min} L_{i}(X)$ is a maximum. Geometrically this is the problem of having given $n$ opaque hyperplanes $L=L_{i}(X)$ in $p+1$ dimensional space and considering 
the $L$ axis as the up and down axis, to find the highest point an observer below all these hyperplanes can see i.e. the highest point in the closed convex region bounded by $L=\operatorname{Min} L_{i}(X)$. To find this point we will start at an arbitrary point of $\operatorname{Min} L_{i}(X)$ and determine a path, consisting of a finite number of line segments which are always going up and lead us to our destination. ${ }^{1}$

Select any point $X^{1} \equiv\left(x_{1}^{1}, x_{2}^{1}, \cdots, x_{p}^{1}\right)$ in $p$ dimensional Euclidean space. Let our linear forms be labelled so that $L_{1}\left(X^{1}\right)=\operatorname{Min} L_{i}\left(X^{1}\right)$. Any ray $l$ starting at $X^{1}$ may be expressed in the normal form: $x_{j}=x_{j}^{1}+b_{j} s$ with $\sum_{1}^{p} b_{j}^{2}=1, s \geqq 0$. Along $l$ we have $L_{1}(X)=\sum_{1}^{p} a_{1}^{j} x_{j}^{1}$ $+a_{1}^{j} b_{j} s+c_{1}$ and $d L_{1} / d s=\sum_{1}^{p} a_{1}^{j} b_{j}$. By the Cauchy-Schwarz inequality $d L_{1} / d s$ is a maximum when $b_{j}=a_{1}^{j} /\left(\sum\left(a_{1}^{i}\right)^{2}\right)^{1 / 2}$. Let $l_{1}$ denote the ray $x_{j}=x_{j}^{1}+a_{1}^{j} s /\left(\sum\left(a_{1}^{i}\right)^{2}\right)^{1 / 2}$. If $a_{1}^{j}=0$ for $j=1,2, \cdots, p$, i.e. $L_{1}=c_{1}$ then $X^{1}$ is a maximum point (there may be a $p-1$ dimensional manifold of such points). If $a_{1}^{j} \neq 0$ for some $j$ and the ray $l_{1}$ does not contain any points for which $L_{1}(X)=L_{i}(X), i \neq 1$, then $L_{i}(X)>L_{1}(X)$ at all points on $l_{1}$ and there is no point for which $\operatorname{Min} L_{i}(X)$ is a maximum.

If the ray $l_{1}$ does have points for which $L_{1}(X)=L_{i}(X), i \neq 1$, let $X^{2}$ be the point on $l_{1}$ corresponding to the smallest value of $s$ for which this condition is satisfied. Denote by $L_{2}(X)$ the (or one of the linear functions) $L_{i}(X)$ for which $L_{1}\left(X^{2}\right)=L_{2}\left(X^{2}\right) \leqq L_{i}\left(X^{2}\right)$ for $i>2$. $X^{2}$ lies in the $p-1$ dimensional manifold $\pi_{1}$, on which $L_{1}(X)=L_{2}(X)$, determined by the equations

$$
\begin{aligned}
x_{i} & =s_{i} \\
x_{p} & =\left(\sum_{1}^{p-1}\left(a_{1}^{j}-a_{2}^{j}\right) s_{j}+c_{1}-c_{2}\right) /\left(\begin{array}{c}
p \\
a_{2}
\end{array}-a_{1}^{p}\right) .
\end{aligned}
$$

In this manifold

$$
L_{1}=L_{2}=\frac{a_{2}^{p} \sum_{1}^{p-1} a_{1}^{j} s_{j}-a_{1}^{p} \sum_{1}^{p-1} \frac{j}{a_{2} s_{j}+c_{1} a_{2}^{p}-c_{2} a_{1}^{p}}}{a_{2}^{p}-a_{1}^{p}} .
$$

Any ray $l$ in $\pi_{1}$ starting at $X^{2}$ may be written in the normal form: $s_{j}=x_{j}^{2}+b_{j} s, \sum b_{j}^{2}=1, s \geqq 0, j=1,2, \cdots, p-1$. Along $l$,

$$
\frac{d L_{1}}{d s}=\sum_{1}^{p-1} c_{j} b_{j} \text { where } c_{j}=\frac{a_{2}^{p} a_{1}-a_{1}^{p} a_{2}^{j}}{a_{2}^{p}-a_{1}^{p}} .
$$

By the Cauchy-Schwarz inequality $d L_{1} / d s$ is a maximum for $b_{j}$ $=c_{j} /\left(\sum c_{j}^{2}\right)^{1 / 2}$. We denote by $l_{2}$ the ray $s_{j}=x_{j}^{2}+c_{j} s /\left(\sum c_{j}^{2}\right)^{1 / 2}$. If $c_{j}=0$

${ }^{1}$ Suggested by Professor J. F. Paydon, U. S. Naval Academy. 
for $j=1,2, \cdots, p-1$ or $\pi_{1}$ is the level intersection of $L=L_{1}(X)$ and $L=L_{2}(X)$, we investigate the question; "Is there any ray starting at $X^{2}$, along which $L_{1}$ and all other $L_{i}$ for which $L_{1}\left(X^{2}\right)=L_{i}\left(X^{2}\right)$, labelled as $L_{2}, L_{3}, \cdots, L_{k}$ are increasing functions?" If the answer is in the negative $X^{2}$ is a point for which $\min L_{i}(X)$ is a maximum. Let $x_{i}=x_{i}^{2}+b_{i} t$ be any ray emanating from $X^{2}$ then along this ray $d L_{i} / d t=\sum_{j=1}^{k} a_{1}^{j} b_{j}, i=1,2, \cdots, k$. A question equivalent to the above is, "Are the inequalities

$$
\sum_{j=1}^{p} a_{i}^{j} b_{j}>0, i=1,2, \cdots k \text { consistent?” }
$$

The consistency of such a set of inequalities may be determined by applying the existence theorems of Dines or Carver (L. L. Dines, Linear inequalities, Bull. Amer. Math. Soc. (1930) p. 393). If the system (1) is consistent a set of $b_{i}$ satisfying (1) may be found by the method of Dines (L. L. Dines, Systems of linear inequalities, Ann. of Math. vol. 20 (1919) pp. 191-198). Using the $b_{i}$ that satisfy (1) we can find a point $P \neq X^{2}$ on the ray $x_{i}=x_{i}^{2}+b_{i} t$ such that $L_{i}(P) \leqq L_{j}(P)$ for $i=1,2, \cdots, k$ and $j>k$. Min $L_{i}(P)>\operatorname{Min} L_{i}\left(X_{2}\right)$. We now repeat the above procedure starting at $P$ as we did starting at $X^{1}$.

If $c_{j} \neq 0$ for some $j$, then there is a gradient ray $l_{2}$. If there is no point $X^{3}$ on $l_{2}$ for which $L_{1}\left(X^{3}\right)=L_{2}\left(X^{3}\right)=L_{i}\left(X^{3}\right)$ for some $i>2$ then there is no maximum value of $\operatorname{Min} L_{i}(X)$. If there is such an $X^{3}$ let it be the one corresponding to the smallest value of $s$ on $l_{2}$. We call $L_{3}(X)$ the (or a) linear function for which $L_{1}\left(X^{3}\right)=L_{2}\left(X^{3}\right)=L_{3}\left(X^{3}\right)$ $\leqq L_{i}\left(X^{3}\right)$ for $i>3$. Proceed as above in the manifold $\pi_{2}$ in which $L_{1}(X)=L_{2}(X)=L_{3}(X)$ and determine $l_{3}$, etc.

Continuing in this way if $X^{r}$ exists, the manifold $\pi_{r-1}$ in which $L_{1}(X)=L_{2}(X)=\cdots=L_{r}(X)$ (assuming these $r-1$ equations are independent, if not we take a smaller $r$ ) is determined by $x_{i}=s_{i}$, $i=1,2,3, \cdots, p-r+1$ and the $x_{j}, j=p-r+2, p-r+3, \cdots, p$ obtained by substituting $x_{i}=s_{i}, i=1,2, \cdots, p-r+1$ in the $r-1$ equations $L_{1}(X)=L_{2}(X)=\cdots=L_{r}(X)$ and solving for $x_{j}$. Any ray in $\pi_{r-1}$ emanating from $X^{r}$ has the form

$$
s_{j}=x_{j}^{r}+b_{j} s \quad=1,2, \cdots p-r+1, s \geqq 0, \sum b_{j}^{2}=1 .
$$

Let

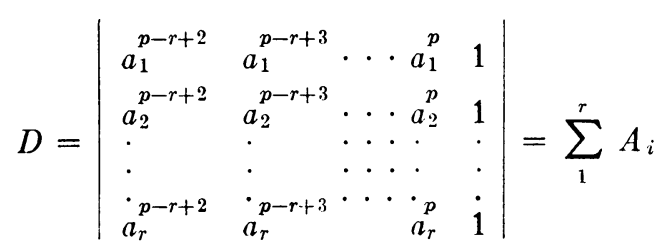


where $A_{i}$ is the cofactor of the $i$ th element in the last column of $D$. Along the ray (2).

$$
\begin{aligned}
& \frac{d L_{1}}{d s}=\sum_{1}^{p-r+1} c_{j} b_{j} \text { where } c_{j}=\sum_{i=1}^{r} \frac{A_{i}^{j} a_{i}}{D} \\
& \frac{d L_{1}}{d s} \text { is a maximum for } b_{j}=c_{j} /\left(\sum c_{j}^{2}\right)^{1 / 2}
\end{aligned}
$$

for $j=1,2, \cdots, p-r+1$. We denote by $l_{r}$ the ray

$$
s_{j}=x_{j}^{r}+c_{j} s /\left(\sum c_{j}^{2}\right)^{1 / 2}
$$

and proceed as we did for $l_{2}$ above.

Since only a finite number of linear functions $L_{i}$ are given, there exist a finite number of level intersections of a subset of the Hyperplanes $L=L_{i}$. Hence if $\operatorname{Max} L_{i}(X)$ exists, its maximum points will lie in a level intersection which will be reached in a finite number of operations as indicated above.

COROLlary. If the inequalities (1) are replaced by

$$
\sum_{j=1}^{p} a_{i}^{j} b_{j} \geqq 0 \quad i=1,2, \cdots, k
$$

and there exist no solutions $\left(b_{1}, b_{2}, \cdots, b_{p}\right)$ of $\left(1^{1}\right)$ other than the trivial solution $b_{j}=0$, then $X^{2}$ or more generally $X^{r}$ is the only point which makes $\min L_{i}(X)$ a maximum. For this implies that there is no direction we can go from $X^{r}$ without some $L_{i}(X)$ decreasing for $i=1,2,3, \cdots$, $r-1$.

The following theorem due to Carver is useful in showing that ( $\left.1^{1}\right)$ has no nontrivial solutions: "A necessary and sufficient condition that the system $\sum_{j=1}^{n} a_{i}^{j} x_{j} \geqq 0, \quad i=1,2, \cdots, m$ admit a solution $\left(x_{1}, x_{2}, \cdots, x_{n}\right)$ which does not annul all the left members is that the adjoint system of linear equations $\sum_{i=1}^{m} a_{i}^{j} y_{i}=0, j=1,2, \cdots, n$ admit no solution $\left(y_{1}, y_{2}, \cdots, y_{m}\right)$ with all the $y_{i}>0$. (Dines, Convex extensions and linear inequalities, Bull. Amer. Math. Soc. vol. 42 (1936) p. 353).

TheOREM II. A maximizing sequence of order $n$ can be obtained in a finite number of operations.

Proof. Express $p_{i}, i=1,2, \cdots, t$ as a product of the $k$ primes $\pi_{1}, \cdots, \pi_{k}$. If $p_{i}=\pi_{1}^{r} \pi_{2}^{s} \cdots \pi_{k}^{\lambda}$ then $s_{i}=r a_{\pi_{1}}+s a_{\pi_{2}}+\cdots+\lambda a_{\pi_{k}}$.

Choosing $a_{1}=0$ we can determine $\Delta_{i}=d_{i}^{1} a_{\pi_{1}}+d_{i}^{2} a_{\pi_{2}}+\cdots+d_{i}^{k} a_{\pi_{k}}$. By setting the expression for $a_{n}$ in terms of $a_{\pi_{1}}, \cdots, a_{\pi_{k}}$ equal to one, 
we can eliminate one of the $a_{\pi_{i}}$ from the $\Delta_{i}$ and obtain $\Delta_{i}$ as a linear combination of $k-1$ of the $a_{\pi_{i}}$. Consider the system of linear inequalities $\Delta_{i}>0$ in terms of the $k-1$ variables $a_{\pi_{i}}$. By Theorem I and the Lemma we can determine a solution of this set of $(t-1)$ inequalities for which $\min \Delta_{i}$ is a maximum.

CoROllary. For every $n$ there exists a maximizing sequence of order $n$ all of whose elements are rational numbers.

MAXimizing SEquences of ORder 2, 3, 4, 5, 6, ANd 7 .

$$
\begin{array}{ll}
n=2, t=3, m_{1}=0 \text { and } m_{2}=1 \text { by property } 1, & \text { Min } \Delta=1 . \\
n=3, t=6, m_{1}=0, m_{2}=2 / 3 \text { and } m_{3}=1, & \text { Min } \Delta=1 / 3 .
\end{array}
$$

Proof. $m_{1}=0, m_{3}=1, t=6$. Let $m_{2}=x$ then

$$
\begin{array}{ll}
s_{1}=0 & \Delta_{1}=x \\
s_{2}=x & \Delta_{2}=1-x \\
s_{3}=1 & \Delta_{3}=2 x-1 \\
s_{4}=2 x & \Delta_{4}=1-x \\
s_{5}=x+1 & \Delta_{5}=1-x \\
s_{6}=2 &
\end{array}
$$

$\Delta_{3}=\Delta_{4}$ for $x=2 / 3$. If $x>2 / 3, \Delta_{4}<1 / 3$. If $x<2 / 3, \Delta_{3}<1 / 3$. Therefore $x=2 / 3$ yields the only maximizing sequence for $n=3$.

$$
n=4, t=9, m_{1}=0, m_{2}=1 / 2, m_{3}=5 / 6, m_{4}=1 ; \quad \min \Delta=1 / 6 \text {. }
$$

Proof. $m_{1}=0, m_{4}=1=2 m_{2}$. Let $m_{3}=y$

$$
\begin{array}{ll}
s_{1}=0 & \Delta_{1}=1 / 2 \\
s_{2}=1 / 2 & \Delta_{2}=y-1 / 2 \\
s_{3}=y & \Delta_{3}=1-y \\
s_{4}=1 & \Delta_{4}=y-1 / 2 \\
s_{5}=y+1 / 2 & \Delta_{5}=1-y \\
s_{6}=3 / 2 & \Delta_{6}=2 y-3 / 2 \\
s_{7}=2 y & \Delta_{7}=1-y \\
s_{8}=y+1 & \Delta_{8}=1-y \\
s_{9}=2 &
\end{array}
$$

$\Delta_{5}=\Delta_{6}$ when $y=5 / 6$ yields the unique maximizing sequence for if $y>5 / 6, \Delta_{7}<1 / 6$ and if $y<5 / 6, \Delta_{6}<1 / 6$. 


$$
\begin{aligned}
n & =5, t=14, \min \Delta=1 / 16 . \\
m_{1} & =0, m_{2}=7 / 16, m_{3}=11 / 16, m_{4}=7 / 8, m_{5}=1 .
\end{aligned}
$$

Proof. $m_{1}=0, m_{5}=1$, let $m_{2}=x, m_{4}=2 x, m_{3}=y$.

$$
\begin{array}{ll}
s_{1}=0 & \Delta_{1}=x=\Delta_{2}+\Delta_{3} \\
s_{2}=x & \Delta_{2}=y-x=\Delta_{9}+\Delta_{4}, y>x \\
s_{3}=y & \Delta_{3}=2 x-y=\Delta_{4}+\Delta_{11}, y<2 x \\
s_{4}=2 x & \Delta_{4}=1-2 x=\Delta_{7}+\Delta_{8}, x<1 / 2 \\
s_{5}=1 & \Delta_{5}=x+y-1, y>1 / 2 \\
s_{6}=x+y & \Delta_{6}=2 x-y=\Delta_{3} \\
s_{7}=3 x & \Delta_{7}=2 y-3 x \\
s_{8}=2 y & \Delta_{8}=x-2 y+1 \\
s_{9}=1+x & \Delta_{9}=x+y-1 \\
s_{10}=2 x+y & \Delta_{10}=1-2 x=\Delta_{7}+\Delta_{8} \\
s_{11}=1+y & \Delta_{11}=4 x-y-1, x>3 / 8 \\
s_{12}=4 x & \Delta_{12}=1-2 x=\Delta_{7}+\Delta_{8} \\
s_{13}=1+2 x & \Delta_{13}=1-2 x=\Delta_{7}+\Delta_{8} \\
s_{14}=2 &
\end{array}
$$

Let $L_{1}=x+y-1, L_{2}=x-2 y+1, L_{3}=4 x-y-1$ and $L_{4}=2 y-3 x$. $L_{2}=L_{3}=L_{4}$ when $x=7 / 16, y=11 / 16$. Applying the corollary to Lemma we consider

$$
\begin{gathered}
x_{1}-2 x_{2} \geqq 0, \quad 4 x_{1}-x_{2} \geqq 0, \quad-3 x_{1}+2 x_{2} \geqq 0 . \\
\left|\begin{array}{rr}
1 & -2 \\
4 & -1 \\
-3 & 2
\end{array}\right|
\end{gathered}
$$

has rank 2 .

The equations $y_{1}+4 y_{2}-3 y_{3}=0$ and $-2 y_{1}-y_{2}+2 y_{3}=0$ are equivalent to $y_{2}=4 / 7 y_{3}$ and $y_{1}=5 / 7 y_{3}$ which obviously has a definite solution. Hence by Carver's theorem $\left(1^{1}\right)$ has no nontrivial solution and $x=7 / 16, y=11 / 16$ determine the unique maximizing sequence.

Similarly we can show that for

$$
n=6, t=18 \text {, } \min \Delta=1 / 23 \text {. }
$$

$m_{1}=0, m_{2}=9 / 23, m_{3}=14 / 23, m_{4}=18 / 23, m_{5}=21 / 23, m_{6}=1$.

And for 


$$
\begin{gathered}
n=7, t=25, \min \Delta=1 / 42 . \\
m_{1}=0, m_{2}=5 / 14, m_{3}=4 / 7, m_{4}=5 / 7, m_{5}=5 / 6, m_{6}=13 / 14, m_{7}=1 .
\end{gathered}
$$

These examples suggest the conjectures: For every $n$ there is a unique maximizing sequence. The $\min \Delta$ of a maximizing sequence is the reciprocal of an integer.

\section{United States Naval Academy and}

The Johns Hopkins University 